\title{
Análise estomática em folhas de Siparuna guianensis Aubl. (Siparunaceae)
}

\author{
Stomatal analysis in leaves of Siparuna guianensis Aubl. (Siparunaceae) \\ Análisis estomático de hojas de Siparuna guianensis Aubl. (Siparunaceae)
}

Recebido: 13/12/2021 | Revisado: 17/12/2021 | Aceito: 22/12/2021 | Publicado: 04/01/2022

\author{
Maria de Fátima Santos \\ ORCID: https://orcid.org/0000-0002-1092-9062 \\ Universidade Federal de Lavras, Brasil \\ E-mail: mariadefatimasmf@gmail.com \\ Natalia Ritter Ruas \\ ORCID: https://orcid.org/0000-0002-1337-4736 \\ Universidade Federal de Lavras, Brasil \\ E-mail: natiiruas@gmail.com \\ Aline Norberto Ferreira \\ ORCID: https://orcid.org/0000-0002-6527-9693 \\ Universidade Federal de Lavras, Brasil \\ E-mail: nf_aline@hotmail.com \\ Marcos Ferrante \\ ORCID: https://orcid.org/0000-0001-6979-2956 \\ Universidade Federal de Lavras, Brasil \\ E-mail: marcos.ferrante@ ufla.br \\ Manuel Losada Gavilanes \\ ORCID: https://orcid.org/0000-0001-7480-6582 \\ Universidade Federal de Lavras, Brasil \\ E-mail: mlgavilanes@ufla.br \\ Elisângela Elena Nunes Carvalho \\ ORCID: https://orcid.org/0000-0002-1124-8066 \\ Universidade Federal de Lavras, Brasil \\ E-mail: elisangelacarvalho@ufla.br
}

\begin{abstract}
Resumo
Os estômatos são importantes estruturas anatômicas presentes principalmente nas folhas das plantas, sendo responsáveis na regulação de trocas gasosas. Estas estruturas são utilizadas na ciência para a identificação de mudanças climáticas sofridas pelo vegetal, além de ser um indicador no controle de qualidade de drogas vegetais. Este trabalho teve como objetivo quantificar e caracterizar os estômatos em folhas de Siparuna guianensis empregando-se dois métodos diferentes de captura de imagens. Para tanto utilizou-se a Microscopia eletrônica de varredura e Microscopia óptica por meio da técnica de impressão da epiderme, sendo avaliados a densidade estomática, índice estomático, diâmetro polar (DP) e equatorial (DE) dos estômatos e a relação entre DP/DE. Foi observado a presença de estômatos na epiderme nas duas faces foliares com predominância na abaxial, permitindo classificar as folhas como anfihipoestomática. Notou-se também diferença significativa nos testes avaliados da densidade estomática entre as faces abaxial e adaxial. Os estômatos desta espécie são classificados com paracítico.

Palavras-chave: Negramina; Anatomia foliar; Estômato; Anfihipoestomática; Impressão da epiderme.
\end{abstract}

\begin{abstract}
The stomata are important anatomical structures present mainly in the plant leaves, being responsible for the regulation of gas exchange. These structures are used in science to identify the climate changes suffered by the plant, as well as being an indicator in the quality control of plant drugs. This work aimed to quantify and characterize the stomata in Siparuna guianensis leaves using two different image capture methods. To do so, scanning electron microscopy and optical microscopy was used, using the epidermis imprinting technique. Stomatal density, stomatal index, polar diameter (PD) and equatorial diameter (ED) of the stomata and the relation between PD/ED were evaluated. The presence of stomas was observed in the epidermis on both leaf faces with predominance on the abaxial side, allowing the leaves to be classified as anfihypoestomatics. There was also a significant difference in the evaluated tests of stomatal density between the abaxial and adaxial faces. The stomata of this species are classified as paracytic.
\end{abstract}

Keywords: Negramina; Leaf anatomy; Stoma; Anfihypoestomatics; Epidermis impression.

\section{Resumen}

Los estomas son importantes estructuras anatómicas presentes principalmente en las hojas de las plantas, siendo responsables de la regulación del intercambio de gases. Estas estructuras se utilizan en la ciencia para identificar los 
cambios climáticos sufridos por la planta, además de ser un indicador en el control de calidad de los medicamentos vegetales. Este trabajo tuvo como objetivo cuantificar y caracterizar los estomas en las hojas de Siparuna guianensis utilizando dos métodos diferentes de captura de imágenes. Para ello, se utilizó la microscopía electrónica de barrido y la microscopía óptica, utilizando la técnica de impresión epidérmica. Se evaluó la densidad estomática, el índice estomático, el diámetro polar (DP) y el diámetro ecuatorial (DE) de los estomas y la relación entre DP/DE. La presencia de estomas en la epidermis se observó en ambas caras de la hoja, con predominio en la abaxial, lo que permitió clasificar las hojas como anfihipostomatica. También se observó una diferencia significativa en las pruebas evaluadas de densidad estomática entre las caras abaxial y adaxial. Los estomas de esta especie se clasifican como paraciticos.

Palabras clave: Negramina; Anatomía de la hoja; Estomas; Anfihipostomatica; Impresión de la epidermis.

\section{Introdução}

Os estômatos são estruturas morfológicas microscópicas, encontradas na camada epidérmica de órgãos vegetais principalmente nas folhas, compostas por duas células estomáticas (células-guarda) que circundam uma abertura, o ostíolo (poro ou fenda). São importantes para a regulação das trocas gasosas da planta, devido à abertura dos ostíolos em resposta as mudanças no ambiente. Os estômatos, além de serem o local responsável pelas trocas de oxigênio e gás carbônico $\left(\mathrm{CO}_{2}\right)$ é também onde ocorre a difusão d'água na transpiração vegetal (Barbosa et al., 2018; Zoulias et al., 2020).

Estas estruturas são bastante sensíveis às variações ambientais, como luz, água, temperatura e a concentração de gás carbônico. A presença de luz, por exemplo, regula positivamente o desenvolvimento estomático, podendo levar à alterações na densidade e índice estomático (Zoulias et al., 2020). Uma baixa densidade estomática restringe a difusão vertical de $\mathrm{CO}_{2}$ por meio da folha e reduz as taxas fotossintéticas, no entanto, a alta densidade estomática provoca a diminuição da difusão de $\mathrm{CO}_{2}$ causando a baixa assimilação de carbono (Simon et al., 2020).

É importante conhecer a morfologia e as características dos estômatos, pois estes permitem ao vegetal otimizar e balancear a fotossíntese como no uso e disponibilidade da água ajustando-se as condições ambientais (Calvo et al., 2020), e na utilização de características epidérmicas das plantas como indicadores de poluição ambiental (Gahlot \& Khanna, 2019), além de ser um parâmetro utilizado para identificar espécies, morfologicamente parecidas, empregadas em medicamentos à base de folhas (Bashir et al., 2020)

Mesmo diante do exposto sobre a importância do conhecimento dos estômatos existem espécies vegetais com escassez de estudos, entre elas a Siparuna guianensis Aubl., (Siparunaceae), um arbusto ou arvoreta aromática, conhecida popularmente como "negramina", "folha-santa", "marinheiro", "limão-bravo" e "capitiư". É uma planta neotropical, sendo encontrada em diferentes domínios fitogeográficos no Brasil, como no bioma Cerrado, Amazônia, Pantanal, Caatinga e Mata Atlântica. As folhas de S. guianensis possuem indicações etnobotânicas/etnofarmacológicas como carminativas, estimulantes, anti-dispépticas, diuréticas, dores no corpo, sinusite, febre, reumatismo, distúrbios inflamatórios e enxaqueca (Almeida et al., 2021; Carvalho et al., 2020; Ferreira et al., 2020; Peixoto et al., 2020; Moura et al., 2020; Valentini et al., 2010).

Devido à escassez de trabalhos morfo-anato-ecológicos realizados com está espécie vegetal, este trabalho teve como objetivo quantificar e caracterizar os estômatos em folhas de Siparuna guianensis.

\section{Metodologia}

Neste trabalho, foram utilizadas duas técnicas de capturas de imagem: microscopia óptica (MO) e microscopia eletrônica de varredura (MEV) em folhas de Siparuna guianensis. O material vegetal utilizado foi identificado pela doutora Ariane Luna Peixoto e o doutor Manuel Losada Gavilanes e uma amostra (exsicata) de material fértil foi depositada no Herbário PAMG, (Herbário da Empresa de Pesquisa Agropecuária de Minas Gerais - EPAMIG, Belo Horizonte, MG), que é considerado herbário fiel depositário, com o número de registro 58647. 
As folhas foram coletadas no período da manhã em Remanescente de Floresta Estacional Semidecidual localizado no Horto de Plantas Medicinais da Escola de Ciências Agrárias de Lavras (ESAL) do Departamento de Agricultura (DAG) da Universidade Federal de Lavras - UFLA ( $21^{\circ} 1^{\prime} 4^{\prime} 42^{\prime \prime}$ S; 45 0' 00" W, 919 metros de altitude). Os experimentos foram realizados no Laboratório de Cultura de Tecidos Vegetais do Departamento de Agricultura - DAG, ESAL/UFLA, no Laboratório de Microscopia Eletrônica e Análise Ultraestrutural do Departamento de Fitopatologia - DFP, ESAL/UFLA e no Laboratório de Anatomia Vegetal do setor de Botânica Estrutural do Departamento de Biologia (DBI) do Instituto de Ciências Naturais ICN/UFLA.

\section{Microscopia óptica (MO)}

Utilizou-se a técnica de impressão da epiderme, em 12 folhas plenamente desenvolvidas, que foram coletadas no $4^{\circ} \mathrm{e}$ $5^{\circ}$ nó, a partir do ápice do ramo; as folhas foram fracionadas, em terço apical, mediano e basal e fixadas em etanol $70 \%$ por 72 horas (Jensen, 1962). Foram obtidas impressões das epidermes das faces adaxial e abaxial, utilizando-se adesivo instantâneo (TEK ${ }^{\circledR}$ BOND); as impressões foram utilizadas para a montagem de lâminas semipermanentes (Segatto et al, 2004) que foram observadas em microscópio de luz (Olympus CX 31, Olympus Optical) acoplado a câmera fotográfica digital (modelo Eurekam 3.0, Bel Engineering).

\section{Microscópia eletrônica de varredura (MEV)}

Foram coletadas 12 folhas plenamente desenvolvidas no $4^{\circ}$ e $5^{\circ}$ nó, a partir do ápice do ramo; foram retirados fragmentos na região mediana entre a nervura primaria e o bordo da lâmina foliar, fixadas em solução de Karnorvsky modificado (2,5\% glutaraldeído, $2 \%$ paraformaldeído em tampão cacodilato de sódio a 0,05M, pH 7,2 e Cloreto de cálcio $\left(\mathrm{CaCl}_{2}\right)$ 0,001 m) por 24 horas. Após esse período, as amostras foram lavadas em cacodilato por 3 vezes, durante 10 minutos e desidratadas em uma série de soluções de acetona (25, 50, 75, 90 e 100\%, este último por 3 vezes) durante 10 minutos. As amostras foram secadas com dióxido de carbono em um secador de ponto crítico (CPD Bal-tec 30), e, em seguida, montadas em suportes (stubs) com fita dupla face de carbono. Posteriormente, foram revestidos com uma camada de ouro de 20 ๆm por evaporação a vácuo (Bal-TEC SDC 50). Os stubs prontos foram observados no microscópio eletrônico de varredura Leo Evo40 XVP (Carl Zeiss, Germany) (Alves \& Perina, 2012).

\section{Análise dos estômatos}

A partir destas técnicas foram obtidas eletromicrografias e fotomicrografias das faces adaxial e abaxial das folhas, sendo mensurados os seguintes parâmetros: diâmetro polar dos estômatos $(\mu \mathrm{m})$; diâmetro equatorial dos estômatos $(\mu \mathrm{m})$; a relação entre diâmetro polar/equatorial (DP/DE); densidade estomática $\left(\mathrm{mm}^{2}\right)$ e porcentagem do índice estomático. As contagens e medições foram feitas utilizando software de análise de imagem ImageJ (National Institutes of Health, Bethesda, Maryland, USA) (Martin et al., 2013) calibrado com régua microscópica fotografada nos mesmos aumentos das fotografias (Gavilanes et al, 2020).

O cálculo do índice estomático foi realizado de acordo com a fórmula: \% Índice Estomático = número de estômatos / (número de estômatos + número de células epidérmicas) X 100 e a Densidade Estomática = estômatos totais $/ \mathrm{mm}^{2}$ (Zoulias et al., 2020).

\section{Estatística}

Os dados obtidos foram submetidos à análise de variância, sendo as médias comparadas pelo teste Scott-Knott com nível de significância de 5\% usando-se o programa Sisvar (Ferreira, 2011). 


\section{Resultados}

As folhas de Siparuna guianensis possuem lâminas foliares simples, de consistência membranácea, de margens lisas, dispostas em filotaxia oposta; pecíolo de 0,5-1,5 cm de comprimento, são alongadas a elípticas ou lanceoladas, com 10,0-22,0 (-30) x 4,0-10,0 (-11) cm, base obtusa, ápice acuminado, inclinado 0,5-1,0 cm de comprimento, a superfície inferior com 9-12 pares de nervuras secundárias levemente salientes (Rennes \& Hausner, 2005), apresentando características visuais que facilitam a diferenciação da face adaxial da abaxial. A superfície adaxial apresenta uma coloração esverdeada mais escura (Figura 1A), enquanto a abaxial é mais clara (Figura 1B).

Figura 1. Superfície adaxial (A) e abaxial (B) da lâmina foliar de Siparuna guianensis.

A

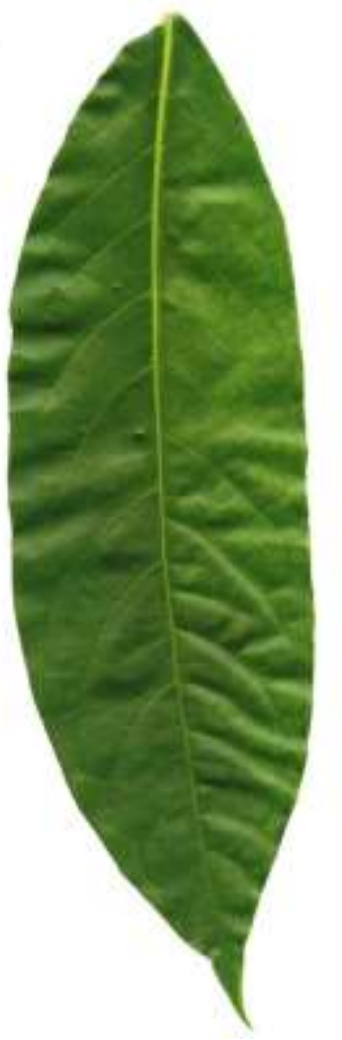

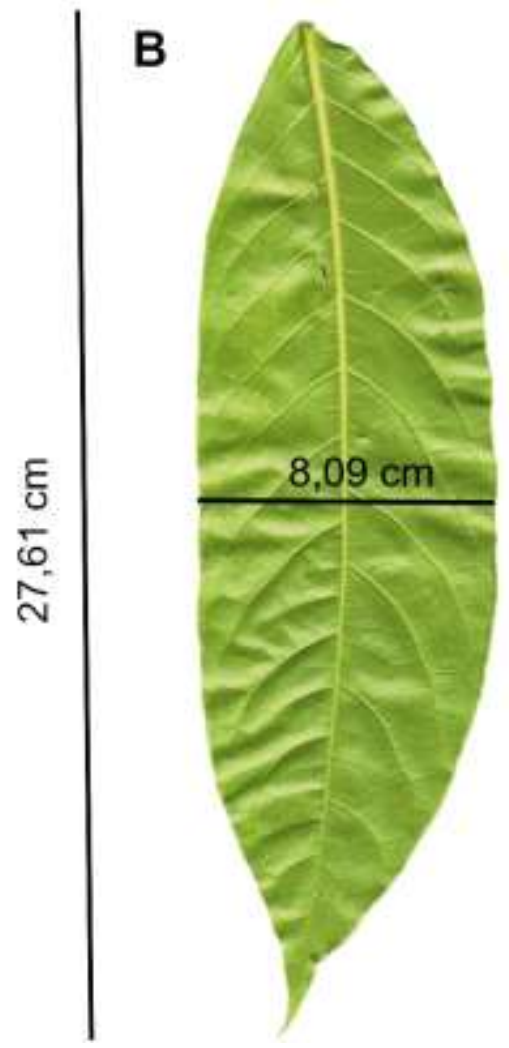

Fonte: Autores (2021).

A Siparuna guianensis, apresenta estômatos na epiderme das duas faces foliares: adaxial (Figura 2A e C) e abaxial (Figura 2B e D), mais frequentes na face abaxial o que permite classificar a folha como anfihipoestomática. Os estômatos são classificados como paracíticos. 
Figura 2. Imagens dos estômatos da folha de Siparuna guianensis.
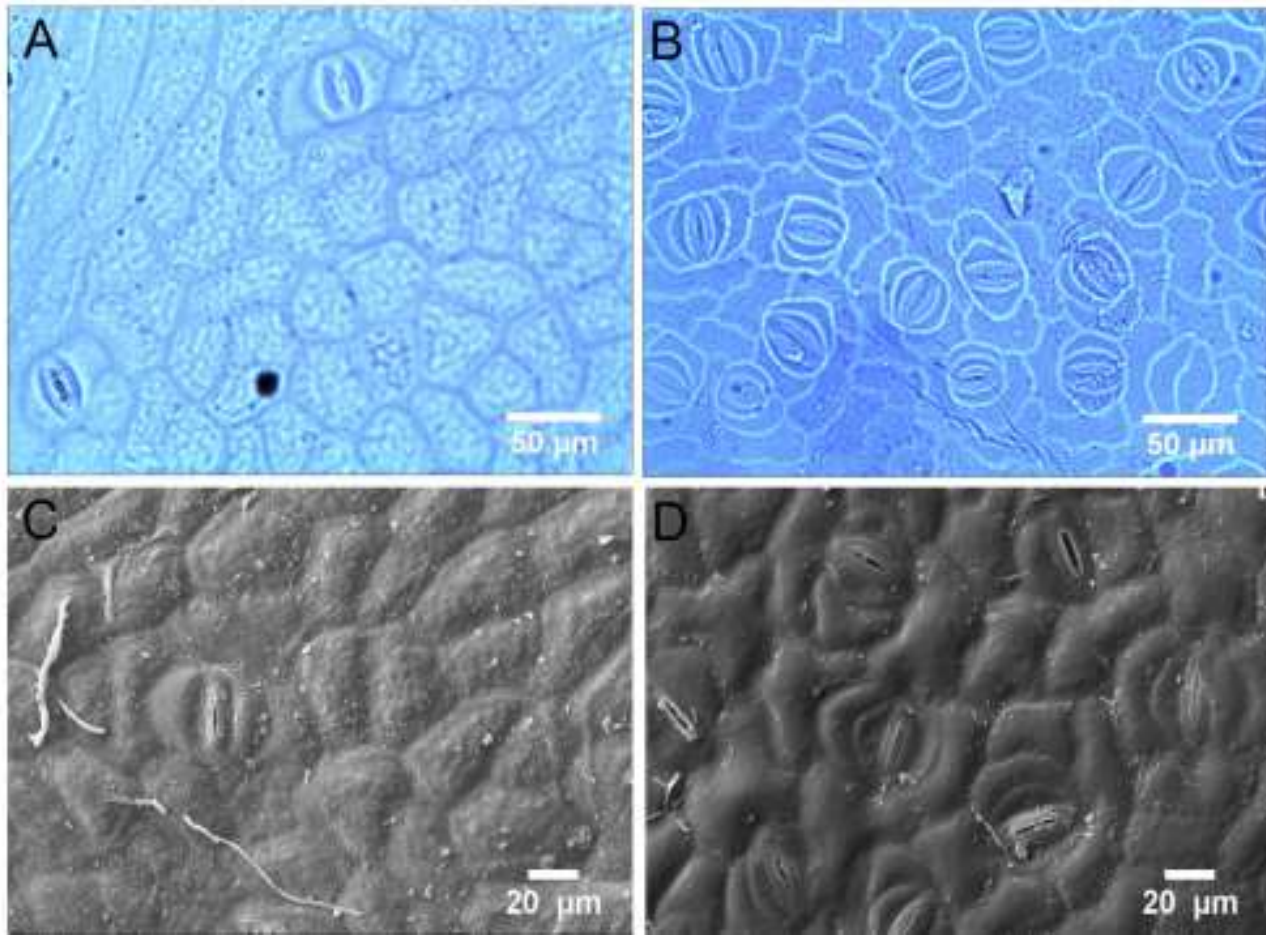

Legenda: Impressões da epiderme - Microscopia Óptica: A. face adaxial, B. face abaxial; Microscopia eletrônica de varredura: C. face adaxial e D. face abaxial. Fonte: Autores (2021).

$\mathrm{Na}$ análise estomática, ambos os testes verificados apresentaram diferenças significativas entre as faces, pois como mencionado anteriormente esta espécie apresenta maior número de estômatos na face abaxial, sendo em média observados 8 a 14 estômatos por área $\left(\mu \mathrm{m}^{2}\right)$; também foi averiguada diferenças entres os métodos de captura, mas isto já era esperado, pois cada microscópio atinge aumentos diferentes. No MEV os estômatos foram avaliados em aumento de 1.000 vezes (apresentado à área da eletromicrografia de $25,52 \mu \mathrm{m}^{2}$ ) e no MO utilizou-se um aumento de 200 vezes (obtendo-se uma área de $12,39 \mu \mathrm{m}^{2}$ na fotomicrografia).

A densidade estomática, que é representada pelo número de estômatos por $\mathrm{mm}^{2}$, exibiu na face abaxial das

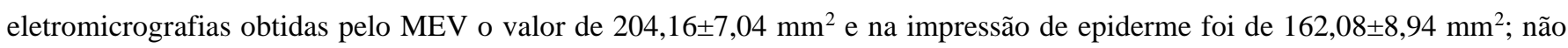
havendo, diferença significativa entre os tratamentos na face adaxial (Tabela 1).

Tabela 1. Densidade estomática, diâmetro polar, equatorial e DP/DE da folha de Siparuna guianensis sobre diferentes técnicas histológicas.

\begin{tabular}{cccccc}
\hline Técnicas histológicas & Face foliar & $\begin{array}{c}\text { Densidade } \\
\text { estomática }\left(\mathbf{m m}^{2}\right)\end{array}$ & $\begin{array}{c}\text { Diâmetro } \\
\text { polar }(\boldsymbol{\mu m})\end{array}$ & $\begin{array}{c}\text { Diâmetro } \\
\text { equatorial }(\boldsymbol{\mu m})\end{array}$ & DP/DE $(\boldsymbol{\mu m})$ \\
\hline MEV & \multirow{2}{*}{ Adaxial } & $27,85 \pm 2,14 \mathrm{c}$ & $22,37 \pm 0,88 \mathrm{c}$ & $14,39 \pm 0,76 \mathrm{c}$ & $1,6 \pm 0,10 \mathrm{a}$ \\
Impressão epidérmica & & $15,48 \pm 1,62 \mathrm{c}$ & $24,54 \pm 0,90 \mathrm{~b}$ & $17,86 \pm 0,59 \mathrm{a}$ & $1,39 \pm 0,07 \mathrm{~b}$ \\
\hline MEV & \multirow{2}{*}{ Abaxial } & $204,16 \pm 7,04 \mathrm{a}$ & $19,57 \pm 0,58 \mathrm{~d}$ & $12,16 \pm 0,39 \mathrm{~d}$ & $1,65 \pm 0,08 \mathrm{a}$ \\
Impressão epidérmica & & $162,08 \pm 8,94 \mathrm{~b}$ & $27,80 \pm 0,75 \mathrm{a}$ & $16,14 \pm 0,29 \mathrm{~b}$ & $1,73 \pm 0,05 \mathrm{a}$ \\
\hline & $\mathrm{CV}(\%)$ & 25,24 & 13,65 & 13,05 & 19,82
\end{tabular}

Média \pm erro padrão, seguidas de mesmas letras na coluna não diferem estatisticamente entre si ao nível de $\mathrm{p} \leq 0,05$ segundo o teste de Scott-Knott. (face abaxial $n=24$ e adaxial $n=12$ ). Fonte: Autores (2021). 
Os diâmetros polar e equatorial (Tabela 1) foram mensurados em estômatos que apresentaram ostíolo fechado. Estas variáveis demostraram resultados diferentes nas faces da folha de Siparuna guianensis, exibindo diâmetro maior nos estômatos

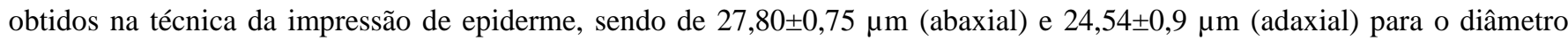

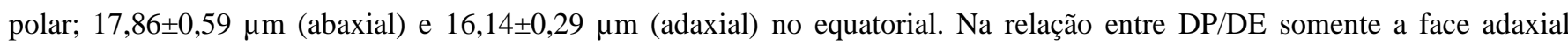
obtida por impressão da epiderme apresentou variação entre os tratamentos $(1,39 \pm 0,07 \mu \mathrm{m})$.

Nas eletromicrografias obtidas no $\mathrm{MEV}$, foi possível realizar a contagem das células comuns da epiderme para calcular a porcentagem de índice estomático (I.E). Já a técnica de impressão da epiderme obtida no MO não foi um método viável para a Siparuna guianensis, pois impossibilitou a visualização nítida das células comuns da epiderme em algumas

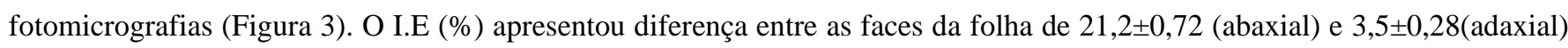
(Gráfico 1).

Figura 3. Estômatos obtido por impressão da epiderme.

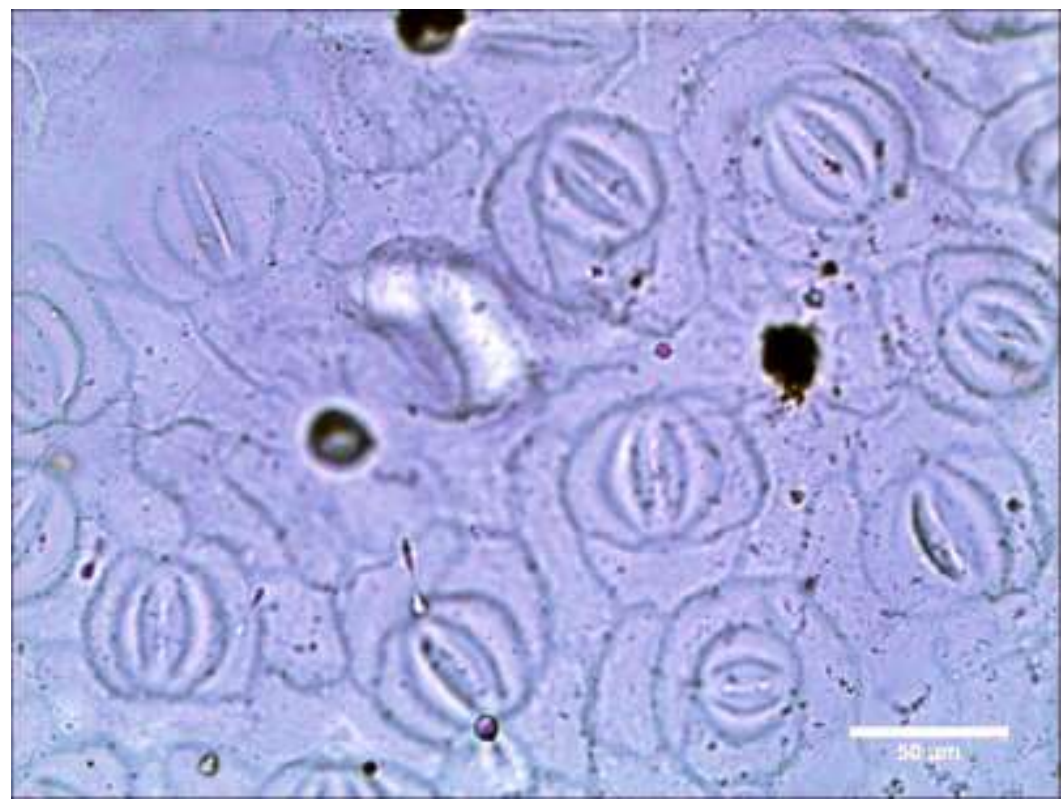

Fonte: Autores (2021).

Gráfico 1. Índice estomático $(\%)$ da região mediana da folha de Siparuna guianensis.

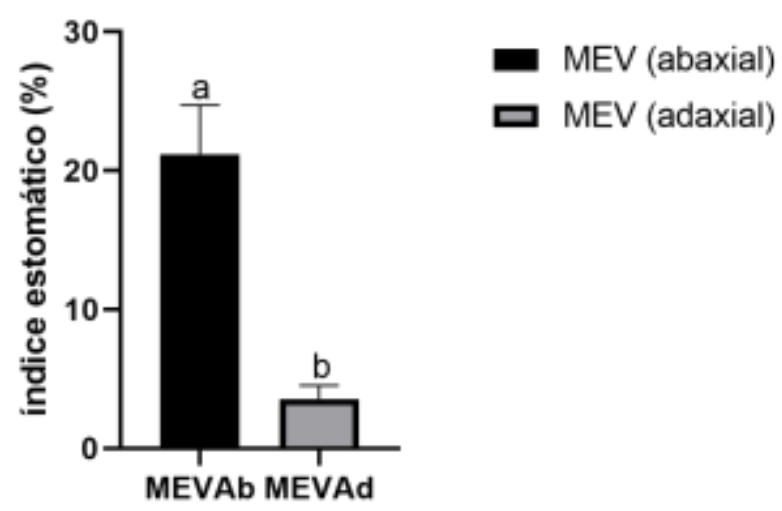

Média seguidas de mesmas letras não diferem estatisticamente entre si ao nível de $\mathrm{p} \leq 0,05$ segundo o teste de Scott-Knott. Fonte: Autores (2021). 
Avaliou-se também a presença de estômatos nas porções ápice, meio e base da folha de Siparuna guianensis em suas diferentes faces, pelo método de impressão da epiderme; os resultados estão demonstrados na Tabela 2. Foi verificada diferença significativa entre as faces foliares, mas não havendo diferença entre os tratamentos na análise da densidade estomática; apresentando somente variações no diâmetro polar na área localizada na base foliar da face abaxial $(30,79 \pm 0,89$ $\mu \mathrm{m})$ e no diâmetro equatorial na base da face adaxial $(19,36 \pm 0,79 \mu \mathrm{m})$.

Tabela 2. Densidade estomática, diâmetro polar, equatorial e DP/DE da folha de Siparuna guianensis pela técnica de impressão da epiderme.

\begin{tabular}{cccccc}
\hline $\begin{array}{c}\text { Região } \\
\text { foliar }\end{array}$ & $\begin{array}{c}\text { Face } \\
\text { foliar }\end{array}$ & $\begin{array}{c}\text { Densidade estomática } \\
\left(\mathbf{m m}^{2}\right)\end{array}$ & $\begin{array}{c}\text { Diâmetro polar } \\
(\boldsymbol{\mu m})\end{array}$ & Diâmetro equatorial $(\boldsymbol{\mu m})$ & DP/DE $(\boldsymbol{\mu m})$ \\
\hline Ápice & & $15,47 \pm 1,62 \mathrm{~b}$ & $22,71 \pm 1,03 \mathrm{c}$ & $17,57 \pm 0,34 \mathrm{~b}$ & $1,29 \pm 0,06 \mathrm{c}$ \\
Meio & Adaxial & $15,48 \pm 1,62 \mathrm{~b}$ & $24,53 \pm 0,90 \mathrm{c}$ & $17,86 \pm 0,59 \mathrm{~b}$ & $1,34 \pm 0,07 \mathrm{c}$ \\
Base & & $15,43 \pm 1,61 \mathrm{~b}$ & $25,82 \pm 1,37 \mathrm{c}$ & $19,36 \pm 0,79 \mathrm{a}$ & $1,38 \pm 0,07 \mathrm{c}$ \\
\hline Ápice & & $167,64 \pm 12,51 \mathrm{a}$ & $28,20 \pm 0,73 \mathrm{~b}$ & $15,21 \pm 0,29 \mathrm{c}$ & $1,87 \pm 0,07 \mathrm{~b}$ \\
Meio & \multirow{2}{*}{ Abaxial } & $162,08 \pm 8,94 \mathrm{a}$ & $27,80 \pm 0,75 \mathrm{~b}$ & $16,14 \pm 0,29 \mathrm{c}$ & $1,73 \pm 0,05 \mathrm{~b}$ \\
Base & & $172,85 \pm 9,39 \mathrm{a}$ & $30,79 \pm 0,89 \mathrm{a}$ & $15,34 \pm 0,34 \mathrm{c}$ & $2,03 \pm 0,08 \mathrm{a}$ \\
\hline & \multirow{2}{*}{$\mathrm{CV}(\%)$} & 35,98 & 14,15 & 10,43 & 17,35 \\
\hline
\end{tabular}

Média \pm erro padrão seguidas de mesmas letras na coluna não diferem estatisticamente entre si ao nível de $p \leq 0,05$ segundo o teste de Scott-Knott. (face abaxial n=24 e adaxial n=12). Fonte: Autores (2021).

\section{Discussão}

As folhas da espécie Siparuna guianensis, apresentam estômatos em ambas a faces foliares abaxial e adaxial, com predominância na face abaxial, sendo a folha classificada com anfihipoestomática. Os estômatos são classificados como paracíticos, denominados desta maneira por apresentarem em torno de sua estrutura duas células subsidiárias (anexas), localizadas de forma que seu eixo longitudinal fica paralelo ao da célula-guarda (Alquini et al., 2006). No trabalho desenvolvido por Bieras e Sajo (2009), realizado por meio de seções transversais da região mediana do ápice foliar e clarificado com hipoclorito de sódio $(20 \%)$ de lâminas da folha de Siparuna guianensis, esses autores verificaram à presença de estômatos somente na face abaxial classificando as folhas como hipoestomáticas, resultado diferente foi obtido neste trabalho, sendo verificado em média 1,25 estômatos por unidade de área nas eletromicrografias da face adaxial.

$\mathrm{Na}$ análise da densidade estomática de acordo Barbosa, Porto e Bertolde (2018), a maior densidade pode estar relacionada quando as folhas são expostas à alta incidência de luz ou quantidade baixa de água, estando relacionado ao controle da condutividade estomática. Isto se justifica porque a Siparuna guianensis apresentou poucos estômatos devido ao ambiente onde ela se encontra um Remanescente de Floresta Estacional Semidecidual, com ausência de irradiação solar diretamente sobre a planta, devido ao sombreamento ocasionado por outras espécies. Este aumento de sombra da planta provoca a diminuição no número de estômatos, mas apresenta aumento na área foliar (Gobbi et al., 2011; Lima Jr. et al., 2006). Diante de alguns trabalhos científicos a Siparuna guianensis é classificada como clímax tolerante à sombra (Nunes et al., 2003; Pinto et al., 2005).

A relação entre os DP e DE fornece um indicativo da forma como é o formato dos estômatos, quanto maior a relação DP/DE, mais elipsoide é seu formato, sendo maior sua função; ao contrário, quanto menor for esta relação menor será seu formato e função (Grisi et al., 2008; Oliveira \& Miglioranza, 2013). Mesmo utilizando métodos diferentes de captura das imagens dos estômatos da Siparuna guianensis, eles apresentaram mesmo formato nas faces abaxial de acordo com a relação DP/DE. 


\section{Considerações Finais}

Os estudos que envolvem caracterização dos estômatos são importantes e auxiliam na identificação das espécies e podem ajudar nos critérios de análise do controle de qualidade de produtos vegetais, considerados medicinais, a base de folhas, além de compreender os impactos das mudanças ambientais na planta.

A Siparuna guianensis é uma espécie anfihipoestomática e os estômatos presentes nas folhas são do tipo paracítico.

Faz se necessário a realizações de mais estudos, futuros, no aspecto botânico da Siparuna guianensis em relação aos tecidos constituintes das suas folhas, da estrutura da venação foliar e de seus frutos, sementes e casca do caule.

\section{Agradecimentos}

À Coordenação de Aperfeiçoamento de Pessoal de Nível Superior (Capes); Conselho Nacional de Desenvolvimento Científico e Tecnológico (CNPq); Fundação de Amparo à Pesquisa do Estado de Minas Gerais (Fapemig) e Universidade Federal de Lavras (UFLA) pela bolsa de estudo; ao Laboratório de Microscopia Eletrônica e Análise Ultraestrutural da UFLA e a doutora Ariane Luna Peixoto por ajudar na identificação da espécie estudada.

\section{Referências}

Almeida, M. A. de., Conegundes, J. L. M., Evagelista, M. de R., Freitas, P. H. de, \& Scio, E. (2021). Avaliação do perfil fitoquímico e do potencial antioxidante do extrato aquoso das folhas de Siparuna guianensis Aublet. Journal of Biology \& Pharmacy and Agricultural Management, 17(2), 252-270.

Alves, E., \& Perina, F. J. (2012). Apostila do curso introdutório à microscopia eletrônica de varredura e microanálise de raios x. UFLA, 63 p.

Alquini, Y., Bona, C., Boeger, M. R. T., Costas, C. G. da, \& Barra, C.F.B. (2006). Epiderme. In: Appezzato-Da-Glória, B., \& Carmello-Guerreiro, S.M. (Ed.), Anatomia Vegetal. UFV (2a ed.) 438p.

Barbosa, L. C., Porto, S. M., \& Bertolde, F. Z. (2018). Análise estomática de duas espécies arbóreas nativas de mata atlântica. Revista Pindorama, 8(8), 1-9.

Bashir, K., Sohail, A., Ali, U., Ullah, A., Ui Haq, Z., Gul, B., Sunera, \& Asghar, M. (2020). Foliar micromorphology and its role in identification of the Apocynaceae taxa. Microscopy Research and Technique, 83(7), 755-766. 10.1002/jemt.23466.

Bieras, A. C., \& Sajo, M. de G. (2009). Leaf structure of the cerrado (Brazilian savanna) woody plants. Trees, 23(3), 451-471. Doi:10.1007/s00468-008-02957 .

Calvo, C. R., Reyes, C. M. P., Armas, I. A., \& Tamargo, A. P. (2020). Characterization of stomata in the species Juglans jamaicensis ssp. insularis (Griseb.) H. Schaarschm. ( walnut tree). Revista Cubana de Ciencias Forestales, 8(1), 122-128.

Carvalho, V. F., Ramos, L. dos A., Silva, C. A. da, Nebo, L., Moraes, D., Silva, F. F. A. da, ... \& Rodrigues, R. M. (2020). In vitro anthelmintic activity of Siparuna guianensis extract and essential oil against Strongyloides venezuelensis. Journal of Helminthology, 94(e50), 1-5. 10.1017/S0022149X19000282.

Ferreira, D. F. (2011). Sisvar: a computer statistical analysis system. Ciência e Agrotecnologia, 35(6), 1039-1042. 10.1590/S1413-70542011000600001.

Ferreira, R. M. dos A., D’Haveloose, N. P., Cruz, R. A. S., Araújo, R. S., Carvalho, J. C. T., Rocha, L., ... \& Souto, R. N. P. (2020). Nano-emulsification enhances the larvicidal potential of the essential oil of Siparuna guianensis (Laurales: Siparunaceae) Against Aedes (Stegomyia) aegypti (Diptera: Culicidae). Journal of Medical Entomology, 57(3), 788-796. 10.1093/jme/tjz221

Gavilanes, M. L., Silva, A. M. da, Dias, M. V. de F., Oliveira, J. A. de, Corrêa. F. F., \& Rodrigues, L. C. de A. (2020). Estrutura foliar de Byrsonima coccolobifolia Kunth. (Malpighiaceae) em ambiente de cerrado e campo rupestre. Rechearch, Society and Development, 9(12), e149912100777. 10.33448/rsdv9i12.10077

Gahlot, L., \& Khanna, V. (2019). Stomatal Index of a few common tree species in the Kishangarh Marble Slurry Dump-yard area, Ajmer (Rajasthan): A case study. Journal of Applied and Natural Science, 11(1), 193-198. 10.31018/jans.v11i1.1977.

Gobbi, K. F., Garcia, R., Ventrella, M. C., Neto, A. F. G., \& Rocha, G. C. (2011). Área foliar específica e anatomia foliar quantitativa do capim-braquiária e do amendoim-forrageiro submetidos a sombreamento. Revista Brasileira de Zootecnia, 40(7) p. 1436-1444. 10.1590/S1516-35982011000700006.

Grisi, F. A., Alves, J. D., Castro, E. M. de, Oliveira, C. de, Biagiotti, G., \& Melo, L. A. (2008). Leaf anatomical evaluations in 'Catuaí' and 'Siriema' coffee seedlings submitted to water stress. Ciência e Agrotecnologia, 32(6), 1730-1736. 10.1590/S1413-70542008000600008.

Jensen, W. A. (1962). Botanical histochemistry. Principles and practices. Freeman, 408 p.

Lima Jr., É. de C., Alvarenga, A. A. de, Castro, E. A. de, Vieira, C. V., \& Barbosa, J. P. R. A. D. (2006). Aspectos fisioanatômicos de plantas jovens de Cupania vernalis camb. submetidas a diferentes níveis de sombreamento. Revista Árvore, 30(1), 33-41. 10.1590/S0100-67622006000100005. 
Research, Society and Development, v. 11, n. 1, e17111124722, 2022

(CC BY 4.0) | ISSN 2525-3409 | DOI: http://dx.doi.org/10.33448/rsd-v11i1.24722

Martin, T. N., Marchese, J.A., Sousa, A. K. F. de, Curti, G. L., Fogolari, H., Santos, V. da C. dos (2013). Uso do software Imagej na estimativa de área foliar para a cultura do feijão. Interciencia: Revista de ciencia y tecnología de América, 38(12), 843-848.

Moura, W. de S., Souza, S. R. de, Campos, F. S., Cangussu, A. S. R., Santos, E. M. S., Andrade, B. S., \& Aguiar, R. W. de S.(2020). Antibacterial activity of Siparuna guianensis essential oil mediated by impairment of membrane permeability and replication of pathogenic bacteria. Industrial Crops and Products, 146, 1-10. 10.1016/j.indcrop.2020.112142.

Nunes, Y. R. F., Mendonça, A. V. R., Botezelli, L., Machado, E. L. M., \& Oliveira-Filho, A. T. de. (2003). Variações da fisionomia, diversidade e composição de guildas da comunidade arbórea em um fragmento de floresta semidecidual em Lavras, MG. Acta Botanica Brasilica, 17(2), 213-229. 10.1590/S010233062003000200005

Oliveira, E. C.de, Miglioranza, É. (2013). Dimensões e densidade estomática em diferentes variedades de mandioca. Cultivando o Saber, 6(4), 201-213.

Peixoto, A. L., Lirio, E. J. \&Pignal, M. (2020). Siparunaceae in Flora do Brasil 2020. Jardim Botânico do Rio de Janeiro.

Pinto, L. V. A., Botelho, S. A., Oliveira-Filho, A. T., \& Davide, A. C. (2005). Estudo da vegetação como subsídios para propostas de recuperação das nascentes da bacia hidrográfica do ribeirão Santa Cruz, Lavras, MG. Revista Árvore, 29(4), 775-793. 10.1590/S0100-67622005000500013.

Renner, S. S.; Hausner, G. (2005). Siparunaceae: Flora Neotropica Monograph 95. New York Botanical Garden, 256p.

Segatto, F. B., Bisognin, D. A., Benedetti, M., Costa, L. C. da, Rampelotto, M. V., \& Nicoloso, F. T. (2004). Técnica para o estudo da anatomia da epiderme foliar de batata. Ciência Rural, 34(5), 1597-1601. 10.1590/S0103-84782004000500042.

Simon, N. M. L., Sugisaka, J., Honjo, M. N., Tunstad, S. A., Tunna, G., Kudoh, H., Dodd, \& A. N. (2020). Altered stomatal patterning accompanies a trichome dimorphism in a natural population of Arabidopsis. Plant Direct, 4(9), 1-6. 10.1002/pld3.262.

Valentini, C. M. A., Rodríguez-Ortíz, C. E., \& Coelho, M. F. B. (2010). Siparuna guianensis Aublet ("negramina"): A review. Revista Brasileira de Plantas Medicinais, 12(1), 96-104. 10.1590/S1516-05722010000100014.

Zoulias, N., Brown, J., Rowe, J., \& Casson, S.A., (2020). HY5 is not integral to light mediated stomatal development in Arabidopsis. Plos One, 15(1), 1-13. 10.1371/journal.pone.0222480. 